\title{
Impact of Sleep Duration on Mortality and Quality of Life in Chronic Kidney Disease: Results from the 2007-2015 KNHANES
}

\author{
Hyo Jin Lee ${ }^{a} \quad N^{2}$ won Kwak ${ }^{a}$ Yong Chul Kim ${ }^{b}$ Sun Mi Choi \\ Jinwoo Lee ${ }^{a, c}$ Young Sik Park ${ }^{a}$ Chang-Hoon Lee ${ }^{a}$ Sang-Min Lee ${ }^{a, c}$ \\ Chul-Gyu Yoo ${ }^{\mathrm{a}, \mathrm{c}}$ Jaeyoung $\mathrm{Cho}^{\mathrm{a}}$
}

${ }^{a}$ Division of Pulmonary and Critical Care Medicine, Department of Internal Medicine, Seoul National University Hospital, Seoul, Republic of Korea; bepartment of Internal Medicine, Seoul National University Hospital, Seoul, Republic of Korea; 'Department of Internal Medicine, Seoul National University College of Medicine, Seoul, Republic of Korea

\section{Keywords}

Sleep duration · Mortality · Quality of life · Chronic kidney disease

\begin{abstract}
Introduction: In the general population, short and long sleep durations have been associated with adverse health outcomes. However, this association remains unclear in patients with chronic kidney disease (CKD). We examined the relationship of sleep duration to mortality and health-related quality of life (HRQOL) in individuals with CKD. Methods: A total of 1,783 adults with CKD who participated in the 2007-2015 Korea National Health and Nutrition Examination Survey were analyzed. CKD was defined as an estimated glomerular filtration rate of $<60 \mathrm{~mL} / \mathrm{min}$ per $1.73 \mathrm{~m}^{2}$. Participants were categorized into 3 groups according to self-reported sleep duration: $<6 \mathrm{~h}$ (short sleepers), 6-8 h, and $>8 \mathrm{~h}$ (long sleepers). The outcome variables were all-cause mortality and HRQOL. HRQOL was assessed using the European Quality of Life-5 Dimensions (EQ-5D) index. Results: During a median of 6.4 years, 481 (27\%) deaths occurred. In unadjusted Cox regression analysis, long sleepers with CKD had an increased risk of death (hazard ratio [HR], 1.62; 95\% con-
\end{abstract}

fidence interval [Cl]: 1.26-2.09). This significant association remained after adjusting for age, sex, and BMI (HR, 1.36; 95\% Cl: 1.05-1.75); however, it was lost after adjusting for CKD stage, social and lifestyle factors, and presence of comorbidities (HR, 1.15; 95\% Cl: 0.89-1.49). Compared with 6- to 8-h sleepers with CKD, long sleepers with CKD had significantly worse HRQOL in multivariable linear regression models. The adjusted means of the EQ-5D index were 0.80 (95\% Cl: $0.77-$ 0.82) for short sleepers, 0.81 (95\% Cl: 0.80-0.82) for 6- to 8-h sleepers, and 0.76 ( $95 \% \mathrm{Cl}: 0.73-0.79)$ for long sleepers ( $p=$ $0.01)$. Discussion/Conclusion: Long sleep duration is associated with poor HRQOL in Korean adults with CKD. The weak association between long sleep duration and mortality was attenuated after multivariable adjustment in this study.

C 2021 The Author(s)
Published by S. Karger AG, Basel

\section{Introduction}

Chronic kidney disease (CKD) is a major public health issue, affecting approximately $10 \%$ of adult populations worldwide, including in Korea [1-3]. CKD is associated with various chronic diseases such as hypertension, type 2 diabetes, and cardiovascular disease [1], resulting in karger@karger.com www.karger.com/ajn

Karger $\stackrel{\text { ' }}{5}$

GOPEN ACCESS
(C) 2021 The Author(s)

Published by S. Karger AG, Basel

This is an Open Access article licensed under the Creative Commons Attribution-NonCommercial-4.0 International License (CC BY-NC) (http://www.karger.com/Services/OpenAccessLicense), applicable to the online version of the article only. Usage and distribution for commercial purposes requires written permission.
Correspondence to:

Jaeyoung Cho, apricot6@snu.ac.kr 
worse health-related quality of life (HRQOL) $[4,5]$ and increased mortality [6, 7]. An effective means of preventing the development and progression of CKD is identifying the lifestyle-related risk factors of an individual [8].

Sleep is essential for optimal health and HRQOL [9]. Substantial evidence suggesting the association between sleep duration and adverse health outcomes in the general population has been accumulating $[8,10]$. Short and long sleep durations have been associated with an increased risk of hypertension, type 2 diabetes, and cardiovascular disease, leading to poor HRQOL and mortality [11-13].

Sleep duration may be a modifiable risk factor for the development and progression of CKD [14]. Previous studies have reported that inadequate sleep duration is associated with an increased risk of CKD development [15-18] and progression to end-stage renal disease (ESRD) $[19,20]$. In addition, several sleep interventions have been applied to enhance HRQOL by improving both the duration and quality of sleep among individuals with CKD $[21,22]$. To improve the understanding of the relationships between sleep duration and adverse health outcomes, especially HRQOL and mortality, in adults with $\mathrm{CKD}$, we conducted this study using data from the Korea National Health and Nutrition Examination Survey (KNHANES) IV to VI.

\section{Materials and Methods}

\section{Study Design and Participants}

This study was based on the KNHANES-linked Cause of Death data (version 1.1). The KNHANES is a nationally representative cross-sectional survey of noninstitutionalized Korean citizens residing in Korea, conducted by the Korea Centers for Disease Control and Prevention (KCDC). The KNHANES is composed of 3-component surveys: health interview, health examination, and nutritional survey. The methodology of the KNHANES has been published in detail [23]. All participants provided written informed consent. The study protocol for the survey was approved by the institutional review board of the KCDC. The current study has been granted an exemption from requiring ethical approval by the Institutional Review Board of the Seoul National University Hospital and was conducted in accordance with the tenets of the Declaration of Helsinki. Among the KNHANES 2007-2015 participants, we enrolled adults ( $\geq 19$ years old) with CKD, which was defined as an estimated glomerular filtration rate (eGFR) of $<60$ $\mathrm{mL} / \mathrm{min}$ per $1.73 \mathrm{~m}^{2}$. Participants who did not agree to the link to death certificate data were excluded.

Variables and Their Definitions

Habitual total sleep duration, including daytime naps, was determined with the following question: "How many hours a day do you usually sleep?" Sleep duration was categorized as $<6,6-8$, and
$>8 \mathrm{~h}$. Short sleepers were defined as those who sleep $<6 \mathrm{~h}$ per day, and long sleepers were defined as those who sleep $>8 \mathrm{~h}$ per day. The eGFR was calculated using the 4-variable Chronic Kidney Disease Epidemiology Collaboration equation, and the categories of glomerular filtration rate were based on the Kidney Disease Improving Global Outcomes 2012 Clinical Practice Guideline for the Evaluation and Management of CKD [24].

The covariates for this study included age, sex, BMI, eGFR, CKD stage, history of tobacco smoking, education, income, living with a spouse, occupation, regular exercise, and comorbidities (e.g., anemia, hypertension, diabetes, cardiovascular disease, cancer, and depression). However, the presence of comorbid sleep disorders, including obstructive sleep apnea (OSA), was not assessed in our study because these data were not collected in the KNHANES. Smokers including former or current smokers were defined as individuals who had smoked $\geq 100$ cigarettes during their lifetime. Education level was classified as middle school or lower and high school or higher. In the KNHANES 2007-2013, regular exercise was defined as $\geq 30$ min of moderate-intensity physical activity for $\geq 5$ days a week or $\geq 20$ min of vigorous-intensity physical activity for $\geq 3$ days a week. In the KNHANES 2014-2015, regular exercise was defined as $\geq 150$ min of moderate-intensity physical activity per week or $\geq 75$ min of vigorous-intensity physical activity per week or an equivalent combination of moderate- and vigorousintensity physical activity ( 1 min of vigorous-intensity physical activity $=2$ min of moderate-intensity physical activity). Data on income (lowest quartile or not), living with a spouse (yes or no), and occupation (yes or no) were also collected.

Anemia was defined as hemoglobin $<12 \mathrm{~g} / \mathrm{dL}$ for nonpregnant women, hemoglobin $<11 \mathrm{~g} / \mathrm{dL}$ for pregnant women, and hemoglobin $<13 \mathrm{~g} / \mathrm{dL}$ for men. Hypertension was defined as systolic blood pressure $\geq 140 \mathrm{~mm} \mathrm{Hg}$ or diastolic blood pressure $\geq 90 \mathrm{~mm} \mathrm{Hg}$ or the use of blood pressure-lowering medication. Diabetes was defined as serum fasting glucose level $\geq 126 \mathrm{mg} / \mathrm{dL}$ or hemoglobin A $1 c \geq 6.5 \%$, or use of antidiabetic medications, or a previous diagnosis of diabetes made by a physician. Cardiovascular disease was defined as angina, myocardial infarction, or stroke as diagnosed by a physician. Participants were considered to have cancer if they had malignant tumors in either of the 6 major organs (stomach, liver, colon, breast, cervix, or lung) diagnosed by a physician. A self-reported diagnosis (made by a physician) of depression was also recorded.

\section{Outcome Variables}

The outcome variables were death of any cause and HRQOL. The dates and causes of death were collected from Statistics Korea. The follow-up duration was calculated from the month and year of the KNHANES to the month and year of death or December 2018, whichever occurred first.

The European Quality of Life-5 Dimensions (EQ-5D) was used to assess HRQOL in the KNHANES. The EQ-5D is a brief, selfcompleted instrument for describing and evaluating the quality of health states and is one of the most widely used instruments for measuring HRQOL [25]. The EQ-5D descriptive system comprises 5 dimensions: (i) mobility, (ii) self-care, (iii) usual activities, (iv) pain/discomfort, and (v) anxiety/depressive mood. For each dimension, participants were asked to select one of the 3 levels of perceived problems (no problems, some problems, and extreme problems) to indicate the most appropriate statement for their health state on the day of the interview. This descriptive profile 
Table 1. Characteristics of adults with CKD according to sleep duration

\begin{tabular}{|c|c|c|c|c|c|}
\hline \multirow[t]{2}{*}{ Characteristic } & \multicolumn{4}{|c|}{ Sleep duration, h/day } & \multirow[t]{2}{*}{$p$ value } \\
\hline & all $(n=1,783)$ & $<6(n=448)$ & $6-8(n=1,123)$ & $>8(n=212)$ & \\
\hline \multicolumn{6}{|l|}{ Age, years } \\
\hline $19-59$ & $183(10)$ & $30(7)$ & $139(12)$ & $14(7)$ & \multirow[t]{4}{*}{$<0.0001$} \\
\hline $60-69$ & $458(26)$ & $92(21)$ & $320(29)$ & $46(22)$ & \\
\hline $70-79$ & $840(47)$ & $237(53)$ & $500(45)$ & $103(49)$ & \\
\hline$\geq 80$ & $302(17)$ & $89(20)$ & $164(15)$ & $49(23)$ & \\
\hline Women & $899(50)$ & $278(62)$ & $521(46)$ & $100(47)$ & $<0.0001$ \\
\hline $\mathrm{BMI}, \mathrm{kg} / \mathrm{m}^{2}$ & $24.4(22.2-26.5)$ & $24.4(22.1-26.7)$ & $24.4(22.3-26.5)$ & $24.1(22.0-26.3)$ & 0.532 \\
\hline eGFR, mL/min per $1.73 \mathrm{~m}^{2}$ & $53(46-57)$ & $53(46-56)$ & $54(47-57)$ & $52(44-56)$ & 0.011 \\
\hline \multicolumn{6}{|l|}{ CKD stage } \\
\hline Stage 3 & $1,677(94)$ & $429(96)$ & $1,055(94)$ & $193(91)$ & \multirow[t]{2}{*}{0.055} \\
\hline Stage $4-5$ & $106(6)$ & $19(4)$ & $68(6)$ & $19(9)$ & \\
\hline Former or current smoker & $852(48)$ & $197(44)$ & $554(49)$ & $101(48)$ & 0.158 \\
\hline Education (high school or higher) & $502(28)$ & $74(17)$ & $377(34)$ & $51(24)$ & $<0.0001$ \\
\hline Income (lowest quartile) & $452(25)$ & $126(28)$ & $274(24)$ & $52(25)$ & 0.296 \\
\hline Living with a spouse & $1,164(65)$ & $240(54)$ & $786(70)$ & $138(65)$ & $<0.0001$ \\
\hline Occupation & $477(27)$ & $102(23)$ & $332(30)$ & $43(20)$ & 0.002 \\
\hline Regular exercise & $314(18)$ & $64(14)$ & $221(20)$ & $29(14)$ & 0.011 \\
\hline Anemia & $519(29)$ & $135(30)$ & $306(27)$ & $78(37)$ & 0.017 \\
\hline Hypertension & $1,303(73)$ & $324(72)$ & $812(72)$ & $167(79)$ & 0.138 \\
\hline Diabetes & $785(44)$ & $192(43)$ & $483(43)$ & $110(52)$ & 0.049 \\
\hline Cardiovascular disease & $367(21)$ & $100(22)$ & 215 (19) & $52(25)$ & 0.118 \\
\hline Cancer & $117(7)$ & $30(7)$ & $70(6)$ & $17(8)$ & 0.623 \\
\hline Depression & $106(6)$ & $33(7)$ & $63(6)$ & $10(5)$ & 0.299 \\
\hline
\end{tabular}

Data are presented as median (interquartile range) or $n$ (\%). eGFR, estimated glomerular filtration rate; CKD, chronic kidney disease.

score can be assigned a summary index score using Korean population-based preference weights $[26,27]$. The EQ-5D index ranges from -0.171 to 1 , with higher values indicating better health status.

\section{Statistical Analysis}

Clinical characteristics were compared between groups using Kruskal-Wallis tests for continuous variables. Categorical variables were compared using either the $\chi^{2}$ test or Fisher's exact test. The amount of missing data ranged from $0 \%$ (age and sex) to $6 \%$ (sleep duration, EQ-5D index, education, and exercise). All missing values were handled using multiple imputations.

Kaplan-Meier survival curves were used to estimate survival and compared using the log-rank test. Cox proportional hazard regression models were constructed to calculate adjusted hazard ratios (HRs) and their 95\% confidence intervals (CIs) for all-cause mortality among short sleepers, 6- to 8-h sleepers, and long sleepers. Four models were constructed. Model 1 included age, sex, and BMI as covariates. Model 2 included CKD stage (stage 3 or stage 4-5) and all covariates in model 1 . Model 3 added socioeconomic characteristics such as history of tobacco smoking, education, income, living with a spouse, occupation, and regular exercise to all covariates in model 2 . The final model (model 4) included the presence of comorbidities such as anemia, hypertension, diabetes, cardiovascular disease, cancer, and depression and all covariates included in model 3. Multiple linear regression models were used to assess the association of sleep duration with the EQ-5D index ad- justed for the same covariates in each model, and the results are presented as linear regression coefficients and 95\% CIs.

Statistical significance was set at $p<0.05$. Statistical analyses were performed using R 4.0.1 software (http://www.r-project.org) and Stata 13.1 software (StataCorp., College Station, TX, USA).

\section{Results}

Of the 1,783 eligible adults with CKD, about half were women. Most participants had stage $3 \mathrm{CKD}$, and the median eGFR was $53 \mathrm{~mL} / \mathrm{min}$ per $1.73 \mathrm{~m}^{2}$. Approximately three-quarters of the participants had hypertension, half had diabetes, and one-fifth had cardiovascular disease. The median sleep duration was $7 \mathrm{~h}$, and short $(<6 \mathrm{~h})$ and long $(>8 \mathrm{~h}$ ) sleepers accounted for 25 and $12 \%$ of the participants, respectively.

The baseline characteristics of the study participants according to sleep duration are listed in Table 1. The proportion of women was higher among short sleepers than among 6- to 8-h sleepers or long sleepers. Participants who had short or long sleep durations were less likely to 


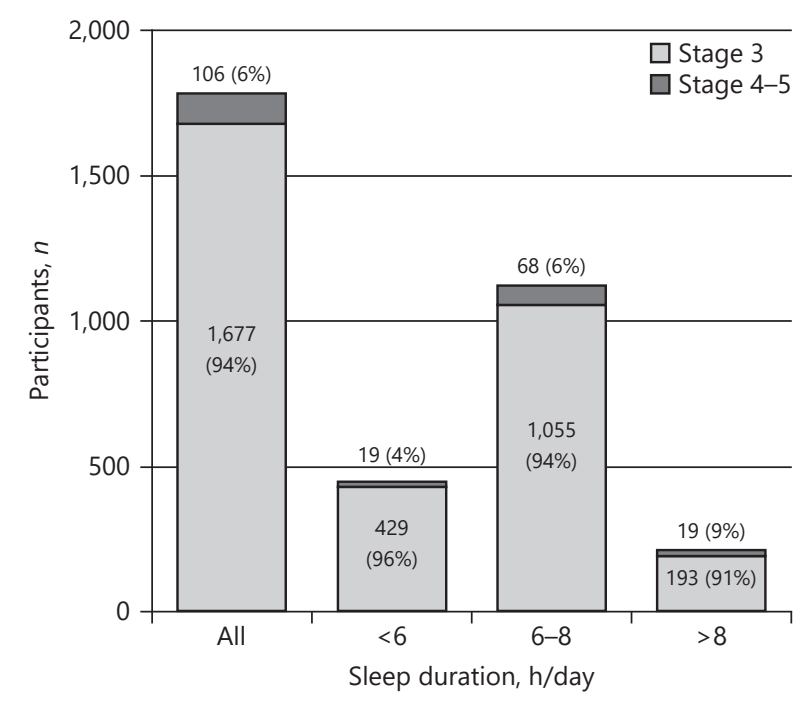

Fig. 1. Distribution of the participants according to stage of CKD. CKD, chronic kidney disease.

have an education of high school or higher, to live with a spouse, to have an occupation, and to exercise regularly than those with a sleep duration of 6-8 h. Anemia and diabetes had the highest prevalence among long sleepers. Participants with stage 4-5 CKD accounted for 4, 6, and $9 \%$ of short, 6-8 h, and long sleepers, respectively (Fig. 1).

During a median follow-up period of 6.4 years (interquartile range [IQR], 4.1-9.0 years), 481 (27\%) deaths occurred. Cardiovascular disease (29\%) was the most common cause of death, followed by cancer (24\%). Figure 2 shows the unadjusted Kaplan-Meier survival curves of all-cause mortality according to sleep duration. In an unadjusted model, long sleepers with CKD had an increased risk of death (HR, 1.62; 95\% CI: 1.26-2.09; Table 2; see online suppl. Table 1; for all online suppl. material, see www.karger.com/doi/10.1159/000516096). This significant association remained in model 1 adjusting for age, sex, and BMI (HR, 1.36; 95\% CI: 1.05-1.75); however, it was lost after adjusting for CKD stage, social and lifestyle factors, and presence of comorbidities (models 2-4; Table 2). In model 4 , the HR for long sleep duration was 1.15 (95\% CI: 0.89-1.49; Table 2). Short sleep duration was not associated with all-cause mortality in the unadjusted and adjusted models (Table 2; online suppl. Table 1). We found no interactions indicating effect modification by age, sex, or presence of comorbidities (online suppl. Table 2).

The median EQ-5D index of the 1,783 participants with CKD was 0.85 (IQR, 0.72-1.00). Compared with participants with CKD with a 6- to 8-h sleep duration, those with a sleep duration of $>8 \mathrm{~h}$ had significantly worse HRQOL in unadjusted and adjusted models $(\beta=-0.05$; $95 \%$ CI: -0.08 to -0.02 in model 4 ; Table 3 ; online suppl. Table 3). However, short sleep duration was not associated with worse HRQOL in adjusted models $(\beta=-0.01$; $95 \%$ CI: -0.04 to 0.01 in model 4 ; Table 3 ). The adjusted means of the EQ-5D index in model 4 were 0.80 (95\% CI: $0.77-0.82$ ) for short sleepers, 0.81 (95\% CI: $0.80-0.82$ ) for 6- to 8-h sleepers, and 0.76 (95\% CI: 0.73-0.79) for long sleepers (Fig. 3). We found no interactions indicating effect modification by age, sex, or presence of comorbidities except cancer (online suppl. Table 4).

\section{Discussion}

In the present study, we found that the significant association between long sleep duration and all-cause mortality was attenuated after controlling for renal function, social and lifestyle factors, and comorbidities in Korean adults with CKD. However, long sleep duration was consistently associated with lower HRQOL, even after multivariable adjustment. Short sleep duration was not associated with all-cause mortality or HRQOL in individuals with CKD.

Many studies have shown a J-shaped association between sleep duration and mortality in the general population: both short and long sleep durations are associated with an increased risk of all-cause mortality $[8,10]$. However, studies examining the association between sleep duration and adverse health outcomes, including mortality in the CKD population, are limited, and most of them have focused on short sleep duration. Previous studies reported that short sleep duration was associated with increased risks of inadequate hydration [28] and CKD development $[15-18,29]$. Other studies reported that short sleep duration in individuals with CKD was associated with progression to ESRD [19, 20], low HRQOL [30], and death [31].

Our study suggests the adverse effects of long sleep duration on health in adults with CKD, which is also consistent with previous research findings. Previous meta-analyses have shown that the association between long sleep duration and all-cause mortality is stronger in older than in younger people and in East Asian countries than in Western countries [32-34]. In addition, results from different large prospective cohorts in South Korea consistently indicated the clinical impacts of long sleep duration: long sleep duration rather than short sleep duration 
Fig. 2. Unadjusted Kaplan-Meier curve for all-cause mortality according to sleep duration.

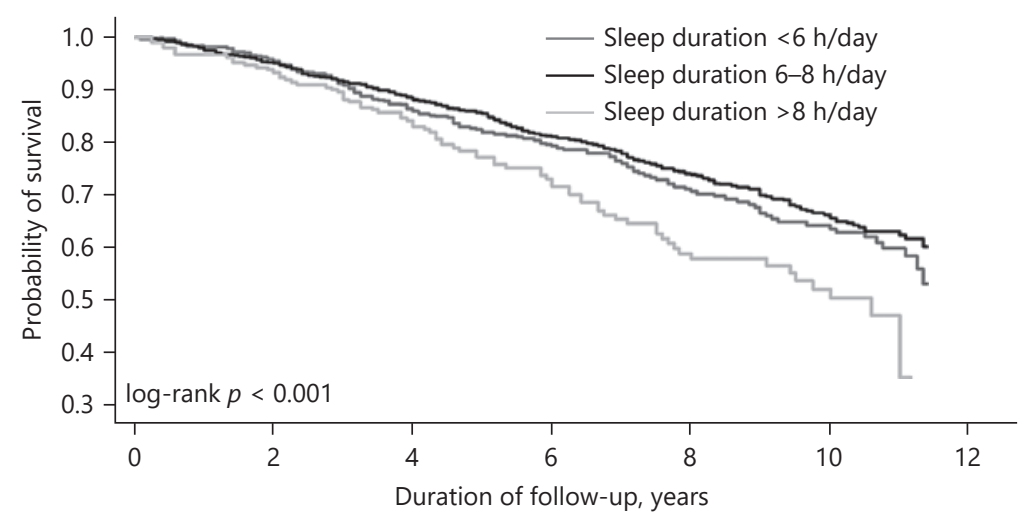

No. at risk

$\begin{array}{cccccccc}<6 & 448 & 430 & 358 & 262 & 155 & 93 & 0 \\ 6-8 & 1,123 & 1,070 & 859 & 614 & 365 & 199 & 0 \\ 8< & 212 & 199 & 154 & 100 & 60 & 31 & 0\end{array}$

Table 2. Multivariable analysis of all-cause mortality according to sleep duration

\begin{tabular}{|c|c|c|c|c|c|c|c|c|c|c|}
\hline \multirow{2}{*}{$\begin{array}{l}\text { Sleep } \\
\text { duration, } \\
\text { h/day }\end{array}$} & \multicolumn{2}{|l|}{ Unadjusted } & \multicolumn{2}{|l|}{ Model 1} & \multicolumn{2}{|l|}{ Model 2} & \multicolumn{2}{|l|}{ Model 3} & \multicolumn{2}{|l|}{ Model 4} \\
\hline & HR $(95 \% \mathrm{CI})$ & $p$ value & HR $(95 \% \mathrm{CI})$ & $p$ value & HR $(95 \% \mathrm{CI})$ & $p$ value & HR $(95 \% \mathrm{CI})$ & $p$ value & $\mathrm{HR}(95 \% \mathrm{CI})$ & $p$ value \\
\hline$<6$ & $1.12(0.91-1.39)$ & 0.273 & $1.02(0.83-1.26)$ & 0.821 & $1.01(0.82-1.25)$ & 0.897 & $0.98(0.79-1.21)$ & 0.849 & $0.97(0.79-1.20)$ & 0.797 \\
\hline $6-8$ & 1.00 (reference) & Reference & 1.00 (reference) & Reference & 1.00 (reference) & Reference & 1.00 (reference) & Reference & 1.00 (reference) & Reference \\
\hline$>8$ & $1.62(1.26-2.09)$ & $<0.0001$ & $1.36(1.05-1.75)$ & 0.019 & $1.26(0.98-1.63)$ & 0.077 & $1.23(0.95-1.59)$ & 0.116 & $1.15(0.89-1.49)$ & 0.278 \\
\hline
\end{tabular}

Data were analyzed with Cox proportional hazard models and are presented as adjusted HR (95\% CI). Model 1: adjusted for age (19-59, 60-69, 70-79, and $\geq 80$ years), sex, and BMI. Model 2: adjusted for all covariates in model 1 plus stage of CKD (stage 3 or stage 4-5). Model 3: adjusted for all covariates in model 2 plus history of tobacco smoking (former or current smoker or never smoker), education (high school or higher or not), income (lowest quartile or not), living with a spouse (yes or no), occupation (yes or no), and regular exercise (yes or no). Model 4: adjusted for all covariates in model 3 plus presence of anemia, hypertension, diabetes, cardiovascular disease, cancer, and depression. CKD, chronic kidney disease; HR, hazard ratio; CI, confidence interval.

was associated with higher urinary albumin excretion in the KNHANES [35] and higher prevalence of CKD in the Korean Genome and Epidemiology Study-Kangwha study [36]. In KNHANES participants, long sleep duration was reported to be associated with sarcopenia [37], which is highly prevalent in patients with advanced CKD [38].

Long sleep duration may have several possible causes. First, impaired quality of nocturnal sleep could result in prolonged sleep duration. Fragmented sleep might not sufficiently reduce sleep pressure and could therefore cause a long sleep duration [39]. Second, comorbid OSA may have an effect on sleep duration. In an epidemiologic study, long sleepers had a greater risk of being diagnosed with OSA [40]. Third, long sleep duration may be a result rather than a cause of comorbidities. Several comorbidities, such as hypertension, diabetes, obesity, and depression, are associated with long sleep duration [41]. Moreover, in patients with CKD, the association between long sleep duration and metabolic comorbidities seems to be modified by comorbid OSA $[42,43]$. In other words, long sleepers may need prolonged sleep because of their comorbid metabolic or mental conditions [39].

Plausible biological mechanisms explaining the association between long sleep duration and adverse health outcomes in the general population have been proposed, including sleep fragmentation, fatigue, change in immune function, photoperiodic abnormalities, lack of physiological challenge, depression, or underlying disease $[32,44]$. In particular, activation of the renin-angiotensin-aldosterone system caused by immune dysfunction can lead to progressive kidney dysfunction [3, 45]. Moreover, long sleep duration combined with habitual late sleep onset is often associated with disturbances in 


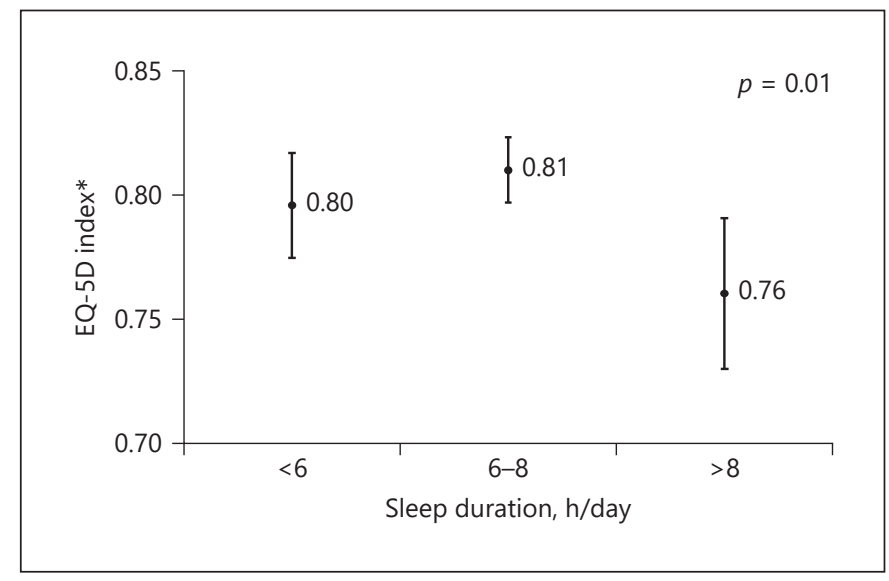

Fig. 3. Adjusted means of the European Quality of Life-5 Dimensions (EQ-5D) index. *Data were analyzed with linear regression with adjustment for the covariates in model 4.

the circadian rhythm [39], which may lead to chronobiological alterations of the renin-angiotensin-aldosterone system [14]. The association of long sleep duration with poor outcomes in individuals with CKD could be explained by potential confounding factors and comorbidities such as sarcopenia, hypertension, and diabetes [11, 41]. This could be the reason for the attenuated association between long sleep duration and all-cause mortality after adjusting for various confounding factors and comorbidities in our study.

Our study had several limitations. First, we relied on self-reported sleep duration, although actigraphy and polysomnography can provide more objective measures. Second, long sleepers accounted for only $12 \%$ of the participants. Third, information on sleep quality and sleep disorders, including OSA and restless legs syndrome, was lacking, as these data were not collected in the KNHANES. OSA may be particularly prevalent in patients with CKD because of nocturnal rostral fluid shift [46]. Fourth, as serum creatinine was measured once in the KNHANES participants, the chronicity of CKD ( $>3$ months) was not examined. Fifth, because most participants had stage 3 $\mathrm{CKD}$, it is difficult to generalize our findings to those with advanced diseases such as ESRD. Sixth, although our study did not find a significant association between short sleep duration and adverse health outcomes in the CKD population, sleep deprivation may remain a considerable problem in this population.

In conclusion, we found that long sleep duration is associated with poor HRQOL in Korean adults with CKD. Although the weak association between long sleep duration and mortality was attenuated after multivariable ad-

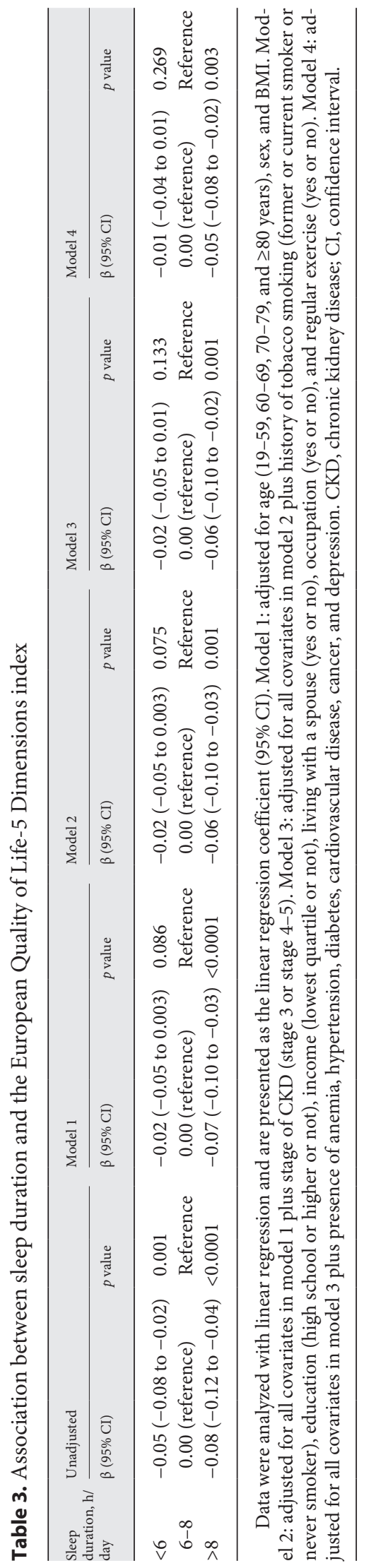


justment in this study, we believe that this finding still supports the importance of the role of nephrology healthcare providers in encouraging adequate sleep duration in patients with CKD. Further large longitudinal studies using objective measures of sleep duration are needed to expand our findings on the effects of sleep duration on adverse health outcomes in the CKD population.

\section{Acknowledgements}

The data in this study were from the Korea National Health and Nutrition Examination Survey, Centers for Disease Control and Prevention, Ministry for Health and Welfare and Cause of Death Statistics, and Statistics Korea.

\section{Statement of Ethics}

The study was approved by the Institutional Review Board (IRB), Seoul National University Hospital.

\section{Conflict of Interest Statement}

The authors have no conflicts of interest to declare.

\section{Funding Sources}

The authors did not receive any funding.

\section{Author Contributions}

All authors (H.J.L., N.K.K., Y.C.K., S.M.C., J.W.L., Y.S.P., C.H.L., S.M.L., C.G.Y., and J.Y.C.) made substantial contributions to the conception or the acquisition, analysis, or interpretation of data for the study. H.J.L. and J.Y.C. drafted the manuscript, and each author provided important intellectual content during revision of the manuscript. All authors approved the final version of the manuscript. All authors agreed to be accountable for all aspects of the work in ensuring that questions related to the accuracy or integrity of any part of the work are appropriately investigated and resolved.

\section{References}

1 Jha V, Garcia-Garcia G, Iseki K, Li Z, Naicker S, Plattner B, et al. Chronic kidney disease: global dimension and perspectives. Lancet. 2013;382(9888):260-72.

2 Park JI, Baek H, Jung HH. Prevalence of chronic kidney disease in Korea: the Korean national health and nutritional examination survey 2011-2013. J Korean Med Sci. 2016; 31(6):915-23.

3 Westhuyzen J, Healy H. Review: biology and relevance of $\mathrm{C}$-reactive protein in cardiovascular and renal disease. Ann Clin Lab Sci. 2000;30(2):133-43.

4 Chow FY, Briganti EM, Kerr PG, Chadban SJ, Zimmet PZ, Atkins RC. Health-related quality of life in Australian adults with renal insufficiency: a population-based study. Am J Kidney Dis. 2003;41(3):596-604.

5 Awuah KT, Finkelstein SH, Finkelstein FO. Quality of life of chronic kidney disease patients in developing countries. Kidney Int Suppl. 2013;3(2):227-9.

6 Matsushita K, Mahmoodi BK, Woodward M, Emberson JR, Jafar TH, Jee SH, et al. Comparison of risk prediction using the CKD-EPI equation and the MDRD study equation for estimated glomerular filtration rate. Jama. 2012;307(18):1941-51.

7 Go AS, Chertow GM, Fan D, McCulloch CE, Hsu CY. Chronic kidney disease and the risks of death, cardiovascular events, and hospitalization. N Engl J Med. 2004;351(13):1296305.

8 Liu TZ, Xu C, Rota M, Cai H, Zhang C, Shi $\mathrm{MJ}$, et al. Sleep duration and risk of all-cause mortality: a flexible, non-linear, meta-regres- sion of 40 prospective cohort studies. Sleep Med Rev. 2017;32:28-36.

9 Watson NF, Badr MS, Belenky G, Bliwise DL, Buxton OM, Buysse D, et al. Recommended amount of sleep for a healthy adult: a joint consensus statement of the American academy of sleep medicine and sleep research society. J Clin Sleep Med. 2015;11(6):591-2.

10 Cai H, Shu XO, Xiang YB, Yang G, Li H, Ji BT, et al. Sleep duration and mortality: a prospective study of 113138 middle-aged and elderly Chinese men and women. Sleep. 2015;38(4): 529-36.

11 Cappuccio FP, Cooper D, D'Elia L, Strazzullo $\mathrm{P}$, Miller MA. Sleep duration predicts cardiovascular outcomes: a systematic review and meta-analysis of prospective studies. Eur Heart J. 2011;32(12):1484-92

12 Jike M, Itani O, Watanabe $\mathrm{N}$, Buysse DJ, Kaneita Y. Long sleep duration and health outcomes: a systematic review, meta-analysis and meta-regression. Sleep Med Rev. 2018;39: 25-36.

13 Itani O, Jike M, Watanabe N, Kaneita Y. Short sleep duration and health outcomes: a systematic review, meta-analysis, and meta-regression. Sleep Med. 2017;32:246-56.

14 Turek NF, Ricardo AC, Lash JP. Sleep disturbances as nontraditional risk factors for development and progression of CKD: review of the evidence. Am J Kidney Dis. 2012;60(5): 823-33.

15 Bo Y, Yeoh EK, Guo C, Zhang Z, Tam T, Chan TC, et al. Sleep and the risk of chronic kidney disease: a Cohort Study. J Clin Sleep Med. 2019;15(3):393-400.
16 Nakajima $\mathrm{H}$, Hashimoto $\mathrm{Y}$, Okamura T, Obora A, Kojima T, Hamaguchi M, et al. Association between sleep duration and incident chronic kidney disease: a population-based cohort analysis of the NAGALA Study. Kidney Blood Press Res. 2020;45(2):339-49.

17 Lin M, Su Q, Wen J, Wei S, Yao J, Huang H, et al. Self-reported sleep duration and daytime napping are associated with renal hyperfiltration in general population. Sleep Breath. 2018;22(1):223-32.

18 Hao Q, Xie M, Zhu L, Dou Y, Dai M, Wu Y, et al. Association of sleep duration with chronic kidney disease and proteinuria in adults: a systematic review and dose-response meta-analysis. Int Urol Nephrol. 2020;52(7): 1305-20.

19 Yamamoto R, Shinzawa M, Isaka Y, Yamakoshi E, Imai E, Ohashi Y, et al. Sleep quality and sleep duration with CKD are associated with progression to ESKD. Clin J Am Soc Nephrol. 2018;13(12):1825-32.

20 McMullan CJ, Curhan GC, Forman JP. Association of short sleep duration and rapid decline in renal function. Kidney Int. 2016; 89(6):1324-30

21 Natale P, Ruospo M, Saglimbene VM, Palmer SC, Strippoli GF. Interventions for improving sleep quality in people with chronic kidney disease. Cochrane Database Syst Rev. 2019; 5(5):Cd012625

22 Ali W, Gao G, Bakris GL. Improved sleep quality improves blood pressure control among patients with chronic kidney disease: a Pilot Study. Am J Nephrol. 2020;51(3):249_ 54. 
23 Kweon S, Kim Y, Jang MJ, Kim Y, Kim K, Choi S, et al. Data resource profile: the Korea national health and nutrition examination survey (KNHANES). Int J Epidemiol. 2014; 43(1):69-77.

24 Levin A, Stevens PE. Summary of KDIGO 2012 CKD Guideline: behind the scenes, need for guidance, and a framework for moving forward. Kidney Int. 2014;85(1):49-61.

25 Brooks R. EuroQol: the current state of play. Health Policy. 1996;37(1):53-72.

26 Nam H, Kim K, Kwon S, Koh K, Poul K. EQ$5 \mathrm{D}$ Korean valuation study using time trade off method. Seoul: Korea Centers for Disease Control and Prevention; 2007.

27 Lee YK, Nam HS, Chuang LH, Kim KY, Yang HK, Kwon IS, et al. South Korean time tradeoff values for EQ-5D health states: modeling with observed values for 101 health states. Value Health. 2009;12(8):1187-93.

28 Rosinger AY, Chang AM, Buxton OM, Li J, $\mathrm{Wu} \mathrm{S}$, Gao X. Short sleep duration is associated with inadequate hydration: cross-cultural evidence from US and Chinese adults. Sleep. 2019;42(2).

29 Petrov ME, Buman MP, Unruh ML, Baldwin CM, Jeong M, Reynaga-Ornelas L, et al. Association of sleep duration with kidney function and albuminuria: NHANES 2009-2012. Sleep Health. 2016;2(1):75-81.

30 Sung SA, Hyun YY, Lee KB, Park HC, Chung W, Kim YH, et al. Sleep duration and healthrelated quality of life in predialysis CKD. Clin J Am Soc Nephrol. 2018;13(6):858-65.

31 Ricardo AC, Goh V, Chen J, Cedillo-Couvert E, Kapella M, Prasad B, et al. Association of sleep duration, symptoms, and disorders with mortality in adults with chronic kidney disease. Kidney Int Rep. 2017;2(5):866-73.
32 Shen X, Wu Y, Zhang D. Nighttime sleep duration, 24-hour sleep duration and risk of allcause mortality among adults: a meta-analysis of prospective cohort studies. Sci Rep. 2016;6: 21480.

33 da Silva AA, de Mello RG, Schaan CW, Fuchs FD, Redline S, Fuchs SC. Sleep duration and mortality in the elderly: a systematic review with meta-analysis. BMJ Open. 2016;6(2): e008119.

34 Cappuccio FP, D’Elia L, Strazzullo P, Miller MA. Sleep duration and all-cause mortality: a systematic review and meta-analysis of prospective studies. Sleep. 2010;33(5):585-92.

$35 \mathrm{Yu} \mathrm{JH}$, Han K, Kim NH, Yoo HJ, Seo JA, Kim SG, et al. U-shaped association between sleep duration and urinary albumin excretion in Korean adults: 2011-2014 Korea National Health and Nutrition Examination Survey. PLoS One. 2018;13(2):e0192980.

36 Choi H, Kim HC, Lee JY, Lee JM, Choi DP Suh I. Sleep duration and chronic kidney disease: the Korean Genome and Epidemiology Study (KoGES)-Kangwha study. Korean J Intern Med. 2017;32(2):323-34.

37 Kwon YJ, Jang SY, Park EC, Cho AR, Shim JY, Linton JA. Long sleep duration is associated with sarcopenia in Korean adults based on data from the 2008-2011 KNHANES. J Clin Sleep Med. 2017;13(9):1097-104.

38 Moorthi RN, Avin KG. Clinical relevance of sarcopenia in chronic kidney disease. Curr Opin Nephrol Hypertens. 2017;26(3):219-28.

39 Tan X, Chapman CD, Cedernaes J, Benedict C. Association between long sleep duration and increased risk of obesity and type 2 diabetes: a review of possible mechanisms. Sleep Med Rev. 2018;40:127-34.
40 Broström A, Sunnergren O, Årestedt K, Johansson P, Ulander M, Riegel B, et al. Factors associated with undiagnosed obstructive sleep apnoea in hypertensive primary care patients. Scand J Prim Health Care. 2012;30(2): 107-13.

41 Patel SR, Malhotra A, Gottlieb DJ, White DP, $\mathrm{Hu} \mathrm{FB}$. Correlates of long sleep duration. Sleep. 2006;29(7):881-9.

42 Sakkas GK, Gourgoulianis KI, Karatzaferi C, Liakopoulos V, Maridaki MD, Pastaka C, et al. Haemodialysis patients with sleep apnoea syndrome experience increased central adiposity and altered muscular composition and functionality. Nephrol Dial Transplant. 2008; 23(1):336-44.

43 Sakkas GK, Karatzaferi C, Zintzaras E, Giannaki CD, Liakopoulos V, Lavdas E, et al. Liver fat, visceral adiposity, and sleep disturbances contribute to the development of insulin resistance and glucose intolerance in nondiabetic dialysis patients. Am J Physiol Regul Integr Comp Physiol. 2008;295(6):R1721-9.

44 Grandner MA, Drummond SP. Who are the long sleepers? Towards an understanding of the mortality relationship. Sleep Med Rev. 2007;11(5):341-60.

45 Yang T, Chou YC, Chu CH, Lin SH, Hsieh $\mathrm{PC}$, Hsu $\mathrm{CH}$, et al. Metabolic syndrome and $\mathrm{C}$-reactive protein concentration as independent correlates of chronic kidney disease. Endocr Res. 2014;39(3):94-8.

46 Yumino D, Redolfi S, Ruttanaumpawan P, Su MC, Smith S, Newton GE, et al. Nocturnal rostral fluid shift: a unifying concept for the pathogenesis of obstructive and central sleep apnea in men with heart failure. Circulation. 2010;121(14):1598-605. 